\title{
EchoGéo
}

49 | 2019

Les corridors de développement

\section{Les corridors industriels en Inde}

Entre libéralisation du capital productif et stratégies de développement régional

\section{Philippe Cadène et Yves-Marie Rault}

\section{OpenEdition}

\section{Journals}

Édition électronique

URL : https://journals.openedition.org/echogeo/17830

DOI : $10.4000 /$ echogeo. 17830

ISSN : 1963-1197

Éditeur

Pôle de recherche pour l'organisation et la diffusion de l'information géographique (CNRS UMR 8586)

Référence électronique

Philippe Cadène et Yves-Marie Rault, « Les corridors industriels en Inde », EchoGéo [En ligne], 49 | 2019, mis en ligne le 23 octobre 2019, consulté le 10 août 2021. URL : http://journals.openedition.org/ echogeo/17830; DOI : https://doi.org/10.4000/echogeo.17830

Ce document a été généré automatiquement le 10 août 2021.

EchoGéo est mis à disposition selon les termes de la licence Creative Commons Attribution - Pas d'Utilisation Commerciale - Pas de Modification 4.0 International (CC BY-NC-ND) 


\title{
Les corridors industriels en Inde
}

\author{
Entre libéralisation du capital productif et stratégies de développement \\ régional
}

Philippe Cadène et Yves-Marie Rault

Cet article a été réalisé dans le cadre des travaux conduits au sein du Groupe de Travail

Circulation des modèles et hétérogénéité des développements du Labex DynamiTe.

\section{Introduction}

1 Les processus contemporains de globalisation ${ }^{1}$, associés à la libéralisation de l'économie à l'échelle mondiale, ont eu deux effets principaux sur les dynamiques d'accumulation spatiale de capital. Tout d'abord, en accélérant et en multipliant les flux de biens, de services, de technologies et de finances, ils ont accru la capacité de certaines régions du monde, davantage encore que des États en leur entier, à capter des formes de capital plus nombreuses et plus diverses (Wallerstein, 2000 ; Arrighi, 2001). Ensuite, en mettant l'ensemble des territoires en concurrence, ces processus globaux ont renforcé les disparités de développement économique entre régions (Harvey, 2008 ; Brenner et al., 2010 ; Sheppard, 2012)

2 Partout dans le monde, et particulièrement au sein des régions à la croissance la plus rapide comme l'Inde, les plus grandes métropoles, bénéficiant des fonctions économiques, politiques, et culturelles majeures, captent la plus grande part du capital $^{2}$. Certaines études ont démontré que la plus forte capacité d'accumulation des grandes villes est largement due à une meilleure connexion aux réseaux mondiaux (Sassen, 1996 et 2002 ; Veltz, 1997). D'autres ont mis en avant leur forte insertion dans un réseau urbain régional comme un aspect central de leur position dominante (Scott, 2001). En tout cas, les réseaux sociaux et économiques, aussi bien que ceux constitués par les infrastructures de transports et communication, apparaissent au cœur des avantages compétitifs des régions dans le contexte de la globalisation (Castells, 1996).

3 Dans la période récente, le développement de corridors économiques à travers le monde semble à la fois confirmer et complexifier ces grilles d'analyse. Territoires encore mal définis, ces corridors se caractérisent pour leur capacité à concentrer le 
long de vastes espaces linéaires connectés aux grandes métropoles et, en même temps, structurant de nouveaux espaces d'accumulation de capital. Ces nouveaux territoires, observés sous des formes diverses tout autour du monde, ont été érigés, à partir du début du nouveau millénaire, en un modèle pour les institutions internationales consacrées au développement (Edginton et al., 2001). La construction de corridors, orientant les investissements vers les infrastructures ou vers les industries, a fait l'objet de nombreux documents d'aménagement territoriaux à l'initiative d'institutions supranationales à l'exemple de la Banque Asiatique de Développement (BAD), et de gouvernements nationaux.

4 En Inde, l'État s'est également engagé depuis les années 2000 dans une politique de développement territorial orientant les investissements le long de corridors où sont implantés des infrastructures de transport modernisées et des zones spécialisées pour héberger des entreprises. Cinq "corridors industriels ou économiques" ont été officiellement identifiés par le gouvernement indien, reliant les plus grandes agglomérations du pays que sont Delhi, Mumbai, Kolkata, Chennai, Bengaluru, et Ahmedabad $^{3}$. Cette stratégie de construction de corridors, où le développement industriel est mis en avant, innove politiquement par son effort pour établir des liens entre différents territoires au sein d'un vaste État fédéral, historiquement clivés par de fortes spécificités culturelles et économiques. Dans un contexte où s'affirme une politique libérale et une ouverture à l'extérieur ayant remis progressivement en cause la stratégie drastique de substitution aux importations décidée par le premier ministre Jawaharlal Nehru au moment de l'Indépendance en 1947, la mise en place des corridors apparaît comme un élément clé de l'intégration territoriale dans un État dont la réussite économique est un enjeu fort pour l'Asie et le monde en son entier. Les succès et les difficultés de la mise en œuvre de cette politique apparaissent donc comme un révélateur de la situation économique dans laquelle se trouve le pays environ trente ans après l'ouverture économique du pays et son entrée dans la globalisation (Boillot, 2016).

5 Cet article souhaite mettre en perspective cette politique indienne des corridors industriels et la dynamique de la répartition sur le territoire du capital productif, que nous définissons ici comme l'ensemble des investissements, biens et services industriels mobilisés afin de générer un profit. Le but de l'analyse est moins de faire un bilan du développement des corridors que de contribuer à une réflexion déjà engagée sur les dynamiques d'organisation du territoire en Inde (Durand-Dastès et al., 1995; Chakravorty et Lall, 2007 ; Chakravorty, 2012 ; Cadène, 2015 ; Shaban et Sanga, 2017). Il s'agit d'abord de faire voir combien, dans un contexte de croissance forte et soutenue, se combinent deux phénomènes. Le premier correspond à la poursuite d'une polarisation de l'économie du pays dans les principales métropoles et dans les grandes régions qu'elles commandent. Le second phénomène réside dans l'affirmation d'une dynamique apparue au tournant du millénaire, le développement de corridors le long desquels se diffusent les investissements et s'organisent les activités de production, renforçant les relations entre les plus grandes métropoles du pays et un certain nombre de villes de tailles diverses, ainsi que les campagnes qui leur sont proches. Il s'agit ensuite de montrer comment la localisation de ces corridors sur le territoire indien ne recoupe qu'en partie les projets gouvernementaux. Ce phénomène illustre combien la libéralisation de l'économie indienne introduit une dynamique de développement que l'État central, en retrait depuis près de trois décennies, peine à contrôler, alors que les logiques d'implantation des entreprises obéissent à des déterminants complexes, 
éloignés de celles des décideurs publics. Cet article participe ainsi d'une réflexion sur le rôle de l'État dans l'orientation de l'économie et l'aménagement du territoire, alors que la globalisation tend à considérablement complexifier les forces en action au sein des sociétés. Émergent ainsi de nouveaux acteurs, beaucoup prenant leurs forces à l'extérieur des territoires concernés, conduisant les États à ne plus décider seuls. Une gouvernance se met en place, l'État partageant les décisions avec des institutions publiques régionales et les sociétés civiles, au premier rang desquelles se trouvent les entrepreneurs et leurs organisations (Strange, 1996; Milbert, 2007; Corbridge et al., 2005).

6 La recherche repose sur l'analyse de la littérature scientifique publié sur le thème. Elle s'appuie également sur une longue pratique de la recherche dans diverses régions indiennes, enrichis de travaux d'enquêtes spécifiques. Celles-ci ont été menées au cours de la décennie 2010 dans plusieurs États indiens ${ }^{4}$, et ont permis la conduite de nombreux entretiens auprès de responsables politiques et administratifs et d'entrepreneurs dans les espaces visités. La réalisation d'un ensemble de cartes, à partir de données de localisation des sièges sociaux des entreprises industrielles et des unités de production, joue enfin un rôle essentiel. Ce travail de cartographie est conduit depuis 1992 et a permis diverses publications sur l'organisation économique du territoire indien (Durand-Dastès et al., 1995 ; Cadène, 2008). Les cartes fournies dans l'article ont été cependant réalisées spécifiquement pour cette recherche. Elles sont le fruit de l'analyse de données recueillies par le Centre for Monitoring Indian Economy (CMIE), un institut privé d'intelligence économique basé à Mumbai ${ }^{5}$. Les informations chiffrées sur la localisation des grandes entreprises indiennes et de leurs unités de production sont très complètes puisqu'elles concernent plus de $85 \%$ du PIB indien. La force et les limites de cette base de données font l'objet d'un commentaire au sein de l'article.

\section{La stratégie de développement de corridors industriels en Inde}

7 Les concepts de «corridor économique » et de «corridor industriel » entrent dans le vocabulaire des décideurs politiques dans le monde au début des années 1990 (Brunner, 2013) et en Inde au milieu des années $2000^{6}$. Cette introduction constitue un changement majeur des modes de planification du territoire. Cette première partie tente de conceptualiser la mise en place des corridors de développement en Inde comme le passage d'une planification visant à l'équilibre du territoire à une stratégie de développement territorial associant secteur public et privé. La localisation du capital productif en Inde est encouragée dans ces espaces qui sont envisagés comme porteurs de croissance, dans un contexte où l'économie est en cours de libéralisation et de globalisation.

\section{De la planification nehruvienne aux stratégies de développement régional}

8 Les premiers efforts de planification territoriale en Inde doivent sans doute être attribués aux colons britanniques. Ces derniers prévoient le développement des villes portuaires permettant de connecter la périphérie au centre colonial dans le cadre 
d'échanges structurellement inégaux, avec des productions à faible valeur ajoutée en Inde, souvent issues de l'agriculture et de l'extraction minière, et la production de biens transformés au Royaume-Uni. Dans les décennies précédant le départ des colons britanniques, le développement de l'industrie dans le sous-continent avait avant tout pour objectif de soutenir l'économie coloniale fondée sur l'exportation de matières premières au profit des industriels britanniques. La création d'entreprises est alors limitée aux objectifs du pouvoir colonial, en particulier l'extraction de minéraux et la sidérurgie pour les infrastructures britanniques (Rothermund, 2002).

$9 \mathrm{Au}$ sortir de l'Indépendance, la politique économique nehruvienne prévoit un développement industriel sur l'ensemble du territoire, grâce à une politique de planification des investissements, tant étatiques que privés. Il s'agit aussi de soutenir l'emploi en s'appuyant sur les petites et moyennes entreprises (PME), qui bénéficient alors d'une réglementation protectrice. Dès 1956, l'Industrial Policy Resolution définit une politique d'équilibrage industriel prévoyant des investissements plus forts dans certains États sous-développés de la fédération, comme le Rajasthan ou le Madhya Pradesh, en agrandissant dans le même temps la liste des produits réservés aux PME. Les plans quinquennaux qui se succèdent visent aussi à améliorer les infrastructures économiques et font émerger une multitude de petits centres économiques à travers le pays.

10 Les réformes libérales commencent au milieu des années 1980, puis s'affirment à partir du début des années 1990 (Corbridge et Harriss, 2000 ; McCartney, 2009; Vakulabharanam et De, 2016 ; Chaudhuri, 2015 ; Boillot, 2016). L'ouverture du pays à la compétition mondiale bouleverse les activités industrielles dans tout le pays et favorisent finalement le développement économique des grandes métropoles indiennes, où se trouvent déjà les plus grandes entreprises et la main d'œuvre qualifiée, capables d'exporter des biens et services à travers le monde. Un État indien volontariste continue de pousser la libéralisation de l'économie dans les années 2000 et déclenche avec prudence l'ouverture de son marché aux capitaux étrangers, tandis que l'objectif consiste à renforcer ses exportations à travers l'amélioration des infrastructures et de l'environnement des affaires.

11 La planification économique et spatiale est alors progressivement remplacée par une stratégie de développement qui passe par la mise en place de territoires attractifs pour les investissements. Sont ainsi créées des Zones Économiques Spéciales (ZES), enclaves juridiques, bénéficiant d'avantages fiscaux et de facilités règlementaires, mais aussi économiques, permettant aux entreprises agglomérées des externalités positives et des économies d'échelle (Dey et Ray, 2011). S'il existe déjà des zones franches industrielles d'exportation en Inde depuis les années 1960, la première ayant été établie sur le port gujarati de Kandla en 1965, une étape est franchie en 2005 lorsqu'est actée une loi encadrant la création de ZES. Les zones franches dont le nombre stagnait, se multiplient rapidement. Alors qu'elles n'étaient que 18 avant 2005, elles sont, sous le régime des ZES, 231 à être opérationnelles en janvier 2019. Ce chiffre illustre combien la politique des ZES a finalement encouragé une concurrence entre les États de la fédération (Kennedy, 2015), voire pour certains auteurs a accordé des privilèges aux entrepreneurs s'y installant, qui évitent ainsi d'investir dans l'amélioration de la productivité de leurs entreprises, grâce à des exemptions fiscales et à des terres obtenues à bas prix, le plus souvent dans le cadre de baux emphytéotiques avantageux (Sharma, 2009). Les États de la fédération les plus industrialisés ont ainsi pu établir le 
plus grand nombre de ZES en mettant en place des législations favorables via leurs propres lois d'encadrement, amendant aussi les lois municipales pour assurer l'autonomie de leurs enclaves économiques (Dey, 2012). Aussi, en 2019, 73 \% des zones opérationnelles du pays étaient situées dans seulement six États: le Tamil Nadu, le Karnataka, le Maharashtra, le Telangana, le Gujarat, et l'Andhra Pradesh.

C'est également en 2005 que se met en place un projet massif de modernisation des infrastructures urbaines, le Jawaharlal Nehru National Urban Renewal Mission, prévoyant un investissement de 18 milliards d'euros sur sept ans. Mais seules les villes de plus d'un million d'habitants sont alors éligibles ${ }^{7}$, et parmi elles, seules trois villes, Visakhapatnam, Surat, et Pune, parviendront à mettre en place l'ensemble des réformes urbaines prévues par le projet. Un seul État, le Gujarat, réussit à faire ces réformes sur l'ensemble de son territoire. La politique de modernisation urbaine est relancée récemment par le gouvernement indien à travers la politique de création de Smart Cities, qui prévoit à partir 2015 le développement de 98 d'entre elles. Le choix consiste cette fois de s'appuyer plus fortement sur les acteurs privés pour la gouvernance et le financement de ces villes nouvelles -souvent d'immenses quartiers autonomes en périphérie de grandes agglomérations, et de mettre en concurrence les villes pour l'allocation des fonds budgétaires dédiés (Datta, 2015; Khan et al., 2018; Milbert, 2017).

Illustration 1 - La localisation des grandes villes en Inde

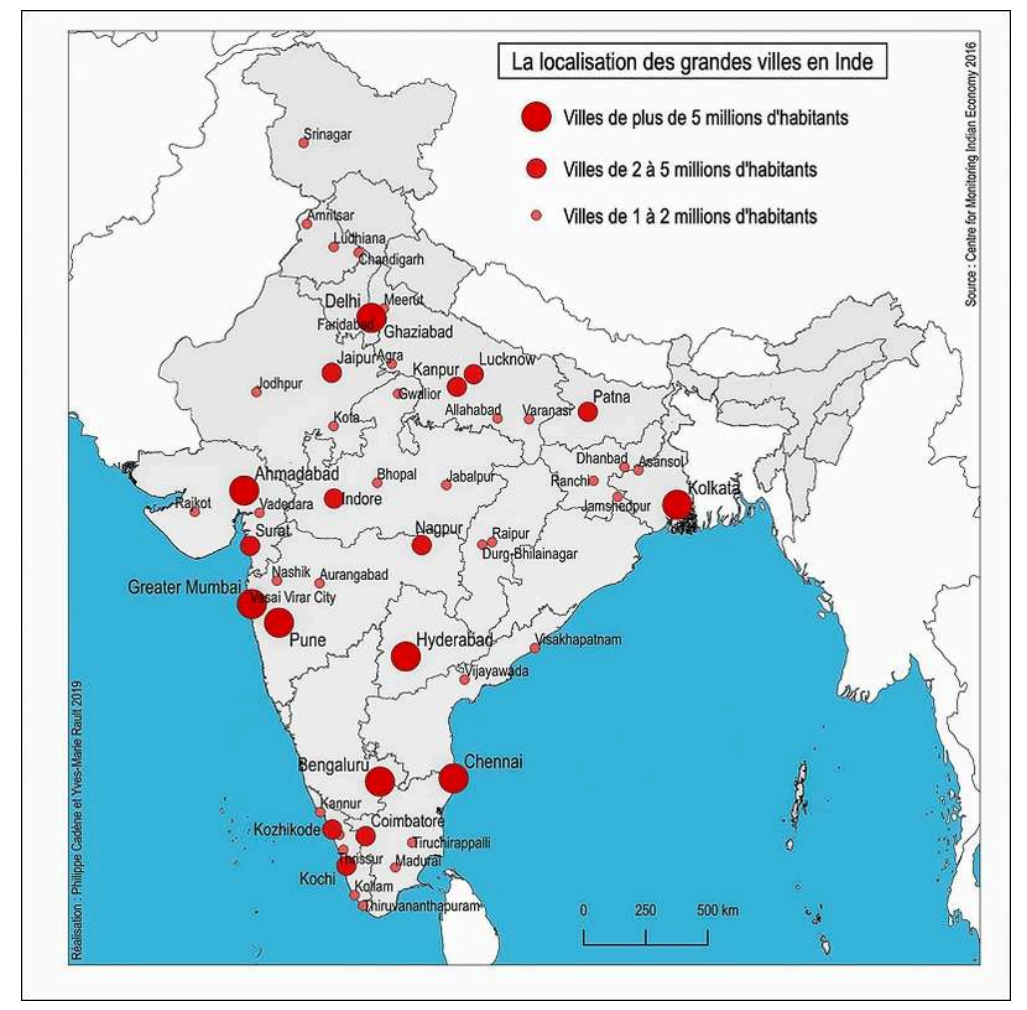

Auteurs : P. Cadène et Y.-M. Rault, 2019.

Initiée dans la deuxième partie des années 2000, la stratégie de développement des corridors indiens s'effectue en synergie avec la politique de développement des ZES et des Smart Cities, les englobant dans des projets de développement plus vastes. Il s'agit d'une étape supplémentaire en direction d'un aménagement dans lequel le pouvoir 
central, devenu progressivement favorable au secteur privé, cherche à attirer les capitaux vers ses grands projets. Les corridors projetés répondent d'ailleurs en Inde aux principes mis en avant par les chercheurs et aménageurs qui envisagent les corridors comme avantageux en matière de rentabilité des capitaux et efficaces pour réduire les coûts d'acheminement des produits d'un hinterland vers les ports (Rodrigue et Notteboom, 2005; Debrie et Comtois, 2010). Plus généralement, la théorie économique voit le développement d'axes de transport, d'infrastructures logistiques, et de zones urbaines comme des phases centrales de la croissance économique d'un corridor, puis d'un territoire national (Srivastava, 2011).

\section{Les cinq corridors définis par le gouvernement indien}

14 Cinq corridors sont définis par le gouvernement indien à partir du milieu des années 2000. Tous prévoient ainsi la construction d'infrastructures de transport et de logistique, la mise en place de régions d'investissement (Investment Regions) et de zones industrielles (Industrial Areas) désignées comme prioritaires. Des projets visant à mettre en place des logements attractifs pour les cadres et les ingénieurs sont aussi prévus avec l'adjonction de villages intégrés (integrated townships) aux zones industrielles et la création de villes nouvelles à la périphérie des plus grandes agglomérations, illustrant les liens existant avec le programme des Smart Cities. La mise en place de chacun des corridors est confiée à une structure ad hoc. En 2017, un organisme gérant l'ensemble des cinq projets, le National Industrial Corridor Development and Implementation Trust (NICDIT), a été créé afin de centraliser la coordination.

Illustration 2 - Les cinq projets de corridors industriels en Inde

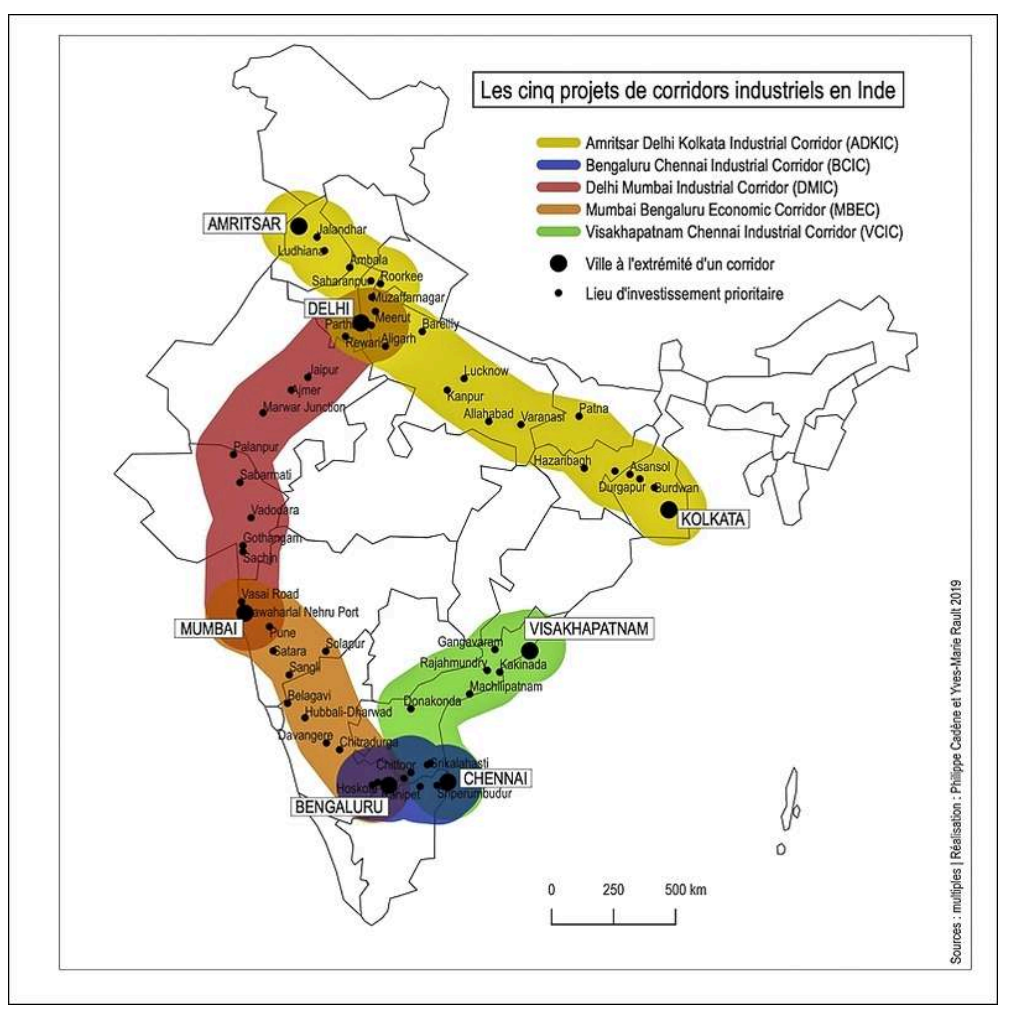

Auteurs : P. Cadène et Y.-M. Rault, 2019. 
Le Delhi-Mumbai Industrial Corridor (DMIC) est le premier qui fut planifié et celui dont le développement est le plus avancé des cinq corridors qui sont mis en place ${ }^{9}$. Il inclut la construction de zones industrielles orientées, de régions d'investissement, de parcs industriels, de ports, d'aéroports, et de centrales électriques. Sept villes nouvelles sont implantées le long du corridor et plusieurs centres logistiques sont prévus. Une institution dédiée, la Delhi Mumbai Industrial Corridor Development Corporation Limited (DMICDC), est mise en place en 2007 avec la collaboration du gouvernement japonais pour lier les deux plus grandes agglomérations indiennes, d'une part, Delhi, la capitale politique, et d'autre part, Mumbai, le cœur économique du pays, intégrant également Ahmedabad, ainsi que plusieurs villes du Gujarat, un des États les plus dynamiques du point de vue industriel. Le projet repose sur de nombreux investissements, publics et privés, qui ont pour but de moderniser ou de construire d'importantes infrastructures de transport et d'encourager le développement de l'industrie et du commerce. Huit aires d'investissements industriels prioritaires sont ainsi définies le long du corridor ${ }^{10}$.

Le gouvernement japonais se place également en soutien d'un second corridor liant Chennai à Bengaluru, sur une distance d'environ $560 \mathrm{~km}$. Le Chennai Bengaluru Industrial Corridor (CBIC), d'ampleur limitée, s'organise autour de trois pôles, Tumkur au Karnataka, Ponneri au Tamil Nadu et Krishnapatnam en Andhra Pradesh, et comporte une trentaine de projets d'investissements priorisant différents secteurs. Il devrait s'articuler autour de deux axes de transport en cours de développement, un réseau ferroviaire dédié aux marchandises et une voie autoroutière.

17 Les troisième et quatrième corridors en cours de mise en place se situent de part et d'autre du CBIC. L'un concerne le lien entre Bengaluru et Mumbai, le Mumbai Bengaluru Economic Corridor (BMEC). Il est soutenu par un accord avec le gouvernement britannique signé en 2013 et accorde une grande importance aux investissements dans les infrastructures de transport, incluant les ports et leur intégration régionale. Projet ambitieux, le BMEC s'organise autour de sept pôles d'investissement prioritaire, Dharwad, Belagavi, Chitradurga-Davangere en Andhra Pradesh et Pune, Satara, Solapur, Sangi au Maharashtra. L'autre corridor s'étend vers le nord à partir de Chennai. Nommé Vizakhapatnam Chennai Industrial Corridor (VCIC), il est, lui, soutenu par l'Asian Development Bank qui a désigné deux pôles spécifiques d'investissements, la ville portuaire de Vishakhapatnam et les villes proches de Srikalahasti et Yerpedu, toutes situées en Andhra Pradesh,

18 Le dernier corridor s'étend sur $1839 \mathrm{~km}$ le long de la plaine indo-gangétique, au nord du pays, le long d'un axe de transport en construction, l'Eastern Dedicated Freight corridor entre Khurja et Mugalsarai, et participe du développement d'un réseau de voies navigables, l'Inland Waterway System, qui s'étend d'Allahabad à Haldia. Nommé Amritsar Delhi Kolkata Industrial Corridor (ADKIC), il correspond à un immense plan d'investissement de long terme concernant les territoires les plus peuplés de l'Inde. Il s'agit d'un projet national élaboré et soutenu par le Ministry of Commerce and Industry qui concerne sept États de la fédération. Chacun d'entre eux est sollicité pour venir en appui d'une vingtaine de grandes villes situées sur le corridor et pour développer au moins une zone industrielle sur leur territoire respectif.

19 L'usage du terme de corridor dans les documents officiels et l'emphase placée sur les investissements dans quelques grands axes de transport ne doivent pas masquer l'ambition de ces projets qui sont tous pensés à des échelles régionales et prennent en compte des territoires s'étendant bien au-delà des axes routiers ou ferroviaires dont ils 
planifient la mise en place. Le corridor Delhi-Mumbai, le projet le plus avancé, en témoigne. Il s'étend sur de très vastes espaces, tant à l'ouest qu'à l'est des axes que constituent l'autoroute $\mathrm{n}^{\circ} 8$ et de la ligne de chemin de fer reliant les deux mégapoles indiennes. Les investissements concernent en effet d'autres itinéraires, tant routiers que ferrés, reliant un vaste hinterland à de nombreux ports, de Kandla, proche de la frontière pakistanaise, à Mumbai, à l'extrémité du corridor. Les industries s'implantent ainsi dans plus de 25 localités, et l'ensemble territorial qui bénéficie du développement s'étend même aux parties les plus reculées du désert du Thar (Cadène, 2017a). Il est ainsi possible d'évoquer l'émergence d'une grande région économique à l'échelle de l'Inde, plutôt qu'un étroit corridor le long duquel s'égrèneraient des investissements (Cadène, 2017b ; Mukhopadhyay, 2018).

\section{La place croissance des acteurs privés dans les projets d'aménagement}

20 La mise en place des corridors en Inde se heurte en fait à des obstacles nombreux. L'insuffisance des infrastructures de transport et de communication est ancienne. Outre de rouler sur des routes congestionnées, les camions eux-mêmes sont très lents et ne peuvent supporter que de faibles tonnages. Dans un contexte où le commerce mondial exige une grande fluidité dans la circulation des biens, le gouvernement s'est lancé depuis le début du nouveau millénaire dans une politique ambitieuse de construction des autoroutes qui manquaient cruellement au pays. Son ambition est de construire « $40 \mathrm{~km}$ d'autoroutes par jour " ${ }^{11}$. Mais depuis le début des années 1980, le budget de l'État s'avère trop faible pour assurer un rythme de financement suffisant ${ }^{12}$.

21 Le secteur privé, national et étranger, a été sollicité, de même que les bailleurs de fonds internationaux comme la Banque Mondiale, sans oublier les gouvernements étrangers. Concernant le financement des corridors, un mécanisme mobilisant des investissements des secteurs publics et privés, avec l'appui des banques multilatérales de développement, est d'ailleurs fortement recommandé par la BAD. Le modèle suivi est donc celui des partenariats public-privé, qui dans les pays asiatiques trouvent souvent un appui d'investisseurs japonais, sud-coréens ou chinois, encadrés par leur gouvernement.

22 Ainsi, les grands projets d'infrastructures de transport dont la réalisation, en Inde comme dans beaucoup d'autres pays, est centrale dans le développement des corridors, repose fortement sur l'appui des acteurs privés. Ces procédures qui existaient déjà dans les années 1990 ont été fortement encouragées par l'État indien à partir de $2012^{13}$. De nombreux appels d'offre ont été lancés par la National Highways Authority of India (NHAI) et ont reçu beaucoup de réponses de la part d'entreprises du secteur qui voient dans ces investissements l'opportunité de récolter d'importantes redevances. Afin de gérer ces projets aux mieux, des institutions ad hoc ont été créées, comme le Dedicated Freight Corridor Corporation of India Limited (DFCCIL), qui est sous la compétence du Ministry of Railways, mais dont le mode de gouvernance intègre les acteurs privés. Ce nouvel intérêt des politiques de développement territorial en Inde pour une gouvernance public-privé apparait déjà clairement dans le cadre de la mise en place des SEZ. Dans l'État du Gujarat, la gestion des SEZ tend par exemple à être dominée par les acteurs privés, bien que le gouvernement cherche à conserver un contrôle en se réservant l'accord de licences d'exploitation. Ces dernières sont progressivement transférées à 
des partenariats public-privé, et ensuite au seul secteur privé. Il en est de même des Smart Cities qui sont financées par des Fonds Communs de Créance (Special Purpose Vehicles) présidé par un directeur général en charge d'attirer les investissements privés et publics et de développer des projets pour les villes concernées.

Cette nouvelle forme de gouvernance impliquant de nombreux acteurs privés ne libère pas le gouvernement des difficultés dans la mise en place effective des corridors. D'une part, des obstacles continuent d'exister, dont tout particulièrement ceux liés aux acquisitions de terres par les gouvernements avant toute opération. Malgré la mobilisation d'une loi coloniale autorisant la saisie de terre par l'État (Dey et Grappi, 2015), la libération du foncier est lente et les délais de réalisation des projets s'allongent. D'autre part, les investissements privés sont libres de s'inscrire ou non dans le cadre de projets gouvernementaux comme ceux des corridors. Au fil des réformes libérales, les entrepreneurs sont devenus maitres des choix des localisations de leurs sièges sociaux et de leurs unités de production. Les territoires industriels de l'Inde ne correspondent donc pas à la géographie envisagée par les décideurs gouvernementaux, fussent-ils acquis aux principes des partenariats public-privé.

\section{Les grandes métropoles indiennes, lieux privilégiés des investissements productifs}

24 Cette partie a pour but de montrer combien, en dépit de nouvelles orientations des politiques publiques, les plus grandes villes indiennes restent au cœur de l'organisation du territoire et rassemblent, pour la presque totalité des secteurs industriels, l'essentiel des capacités de contrôle et des investissements productifs. Cette cartographie repose sur l'analyse de la base de données Prowess concernent les grandes entreprises indiennes fournie par le CMIE. Ces données existent depuis 1989 et s'étendent chaque année. Le présent travail utilise les données au mois de septembre 2016 et concerne 38499 sièges sociaux d'entreprises et les unités de production qui leur sont liées. La base Prowess est constituée après analyse des rapports annuels que fournissent les entreprises, mais aussi des informations provenant des institutions boursières, des agences de notation, sans oublier les sites internet des entreprises et les informations publiées par divers médias. Ainsi en 2009, les entreprises listées représenteraient déjà $84 \%$ du PIB indien et $79 \%$ de la valeur de la production du secteur formel manufacturier. La liste inclut les entreprises privées autant que publiques, ainsi que les coopératives. Elles rassemblent les divers secteurs de la production et des services. N'utilisant que des données générales sur les entreprises, la base constituée pour la réalisation de cet article échappe à la faiblesse majeure de Prowess qui réside dans l'inégale précision des données fournies pour chacune des entreprises.

Les données utilisées dans cet article excluent les entreprises et les unités de production liés aux services et comptabilisent au total 18057 sièges sociaux d'entreprises et 27920 unités de production. Ce choix s'explique par la forte tendance des entreprises de services à se localiser dans les grandes agglomérations. Leur prise en compte aurait ainsi accentué l'effet de polarisation des activités dans ces dernières, au détriment de la visibilité des villes petites et moyennes et donc celle des corridors dans la cartographie. À partir de ces deux ensembles de données, deux cartes ont été réalisées, l'une concernant les sièges sociaux des grandes entreprises industrielles, l'autre les unités de production. Leur présentation structure cette partie. À des fins de 
comparaison, la taille des cercles des deux cartes est établie en référence à une valeur maximale identique afin de conserver une même relation de proportionnalité entre les quantités absolues.

\section{Une forte concentration des sièges sociaux dans les grandes métropoles}

La carte présentant la répartition des sièges sociaux montre la répartition des localités dans lesquelles plus de cinq entreprises sont situées. Elle fait apparaître 186 localités, principalement des métropoles et des villes secondaires, qui sont fortement hiérarchisées. En effet, avec une moyenne de 93 et une médiane de 15, la distribution statistique des sièges sociaux présente une forte asymétrie, avec de nombreuses localités n'ayant qu'un petit nombre de sièges sociaux sur leur territoire et un petit nombre de localités en regroupant un grand nombre.

Illustration 3 - La localisation des sièges sociaux industriels en Inde

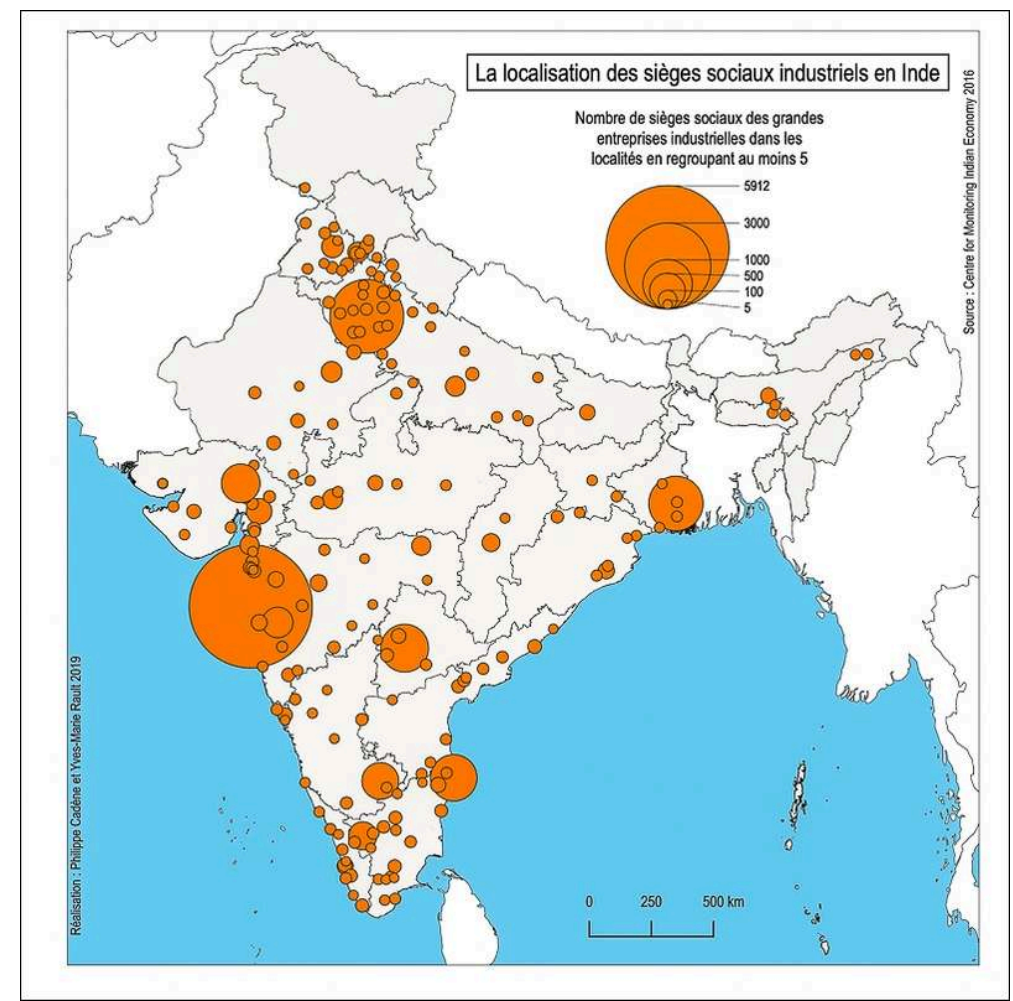

Source : Center for Monitoring Indian Economy, 2016 - Auteurs : P. Cadène et Y.-M. Rault, 2019.

Cette carte illustre combien les deux mégapoles de Mumbai (18 414288 hab. en 2011) et de Delhi (16 314838 hab.) ${ }^{14}$ dominent l'ensemble du territoire et commandent une large part du système économique national. Un tiers des plus puissantes entreprises indiennes, soit 5912 d'entre elles, ont leur siège à Mumbai, tandis que Delhi n'en rassemble que 2 236, occupant toutefois une place importante sur le plan économique. En effet, bénéficiant de son statut de capitale de la fédération indienne, Delhi regroupe, outre l'ensemble des institutions politiques nationales et des institutions internationales ou représentations étrangères, le siège de la plupart des entreprises du secteur public. Les deux plus grandes mégapoles indiennes partagent également des 
institutions culturelles et créatives et semblent être les centres de modernité les plus éminents de l'Inde, voire du sous-continent en son entier. Kolkata, l'ancienne capitale coloniale, (14 $112536 \mathrm{hab}$.), où se trouve également le siège de 1217 grandes entreprises, continue aussi d'exercer une certaine influence sur la dynamique régionale du pays et semble d'ailleurs sortie de la longue crise dans laquelle elle s'était trouvée plongée après l'Indépendance et la partition. Les quatre autres grandes métropoles qui se situent au sommet de la hiérarchie urbaine indienne, Chennai (8 696010 hab.), Bangalore (8 499399 hab.), Hyderabad (7 749334 hab.) et Ahmedabad (6 352254 hab.), tiennent également une grande place sur la carte du fait d'un nombre de sièges sociaux plus importants que les villes suivantes. Il faut alors remarquer que le classement lié au poids démographique de ces grandes villes n'est pas identique à celui du nombre des sièges sociaux d'entreprises. Hyderabad se place en quatrième position avec 936 sièges sociaux, suivie de peu par Chennai qui en rassemble 880. Viennent ensuite Ahmedabad et Bangalore, toutes deux assez proches, avec respectivement 598 et 537 sièges sociaux. Une série de villes de quatrième rang (Cadène, 2012) se distingue également, avec d'abord Pune près de Mumbai, et Coimbatore au sud de Bangalore, puis Vadodara à proximité d'Ahmedabad, Ludhiana, Jaipur, Chandigarh au nord-ouest du pays, Indore, Kanpur, Nagpur dans la partie centrale, et enfin Surat sur le littoral nord de Mumbai.

Il apparaît donc que les entreprises continuent de privilégier les lieux où les investissements sont les plus aisés à rassembler, où une main d'œuvre adéquate est plus facile à trouver, et où se localise la « classe créative » (Florida, 2005) et les institutions éducatives qui les forment, tout particulièrement les établissements scolaires privés prestigieux, les plus grandes universités fédérales ou encore les Indian Institutes of Technology (IIT) et les Indian Institutes of Management (IIM), ces deux derniers ensembles d'institutions d'enseignement supérieur indiennes représentant l'excellence dans le pays. Ce sont aussi ces très grandes villes qui offrent aux entreprises un cadre règlementaire moins contraignant qu'ailleurs en Inde et leur permettent de bénéficier d'infrastructures physiques de meilleure qualité, d'un bon accès aux services bancaires, et d'un ensemble important d'économies d'agglomération, notamment liées à la proximité de secteurs connexes et de fournisseurs. La présence des aéroports internationaux dans ces très grandes villes constitue également un attrait essentiel. Les ressources présentes dans les grandes métropoles indiennes semblent finalement bien plus adéquates au développement de l'entrepreneuriat que le reste des localités du pays (Ghani et al., 2014).

\section{Des lieux de production répartis entre métropoles principales et unités urbaines de diverses tailles}

La seconde carte concernant les lieux de production repose sur le même principe de calcul, soit la prise en compte des villes où plus de cinq unités de production sont localisées, ces dernières appartenant à l'un des sièges sociaux pris en compte dans carte précédente. Elle fait apparaître 688 localités, qui peuvent être des parcs industriels et des villages relativement éloignés des villes aussi bien que des grandes agglomérations urbaines. Avec une moyenne de 33 et une médiane de 11, la distribution statistique des unités de production présente certes une asymétrie moins forte que les sièges sociaux, significative d'une meilleure répartition sur le territoire, mais tend tout de même à confirmer la domination des grands centres urbains du pays. 


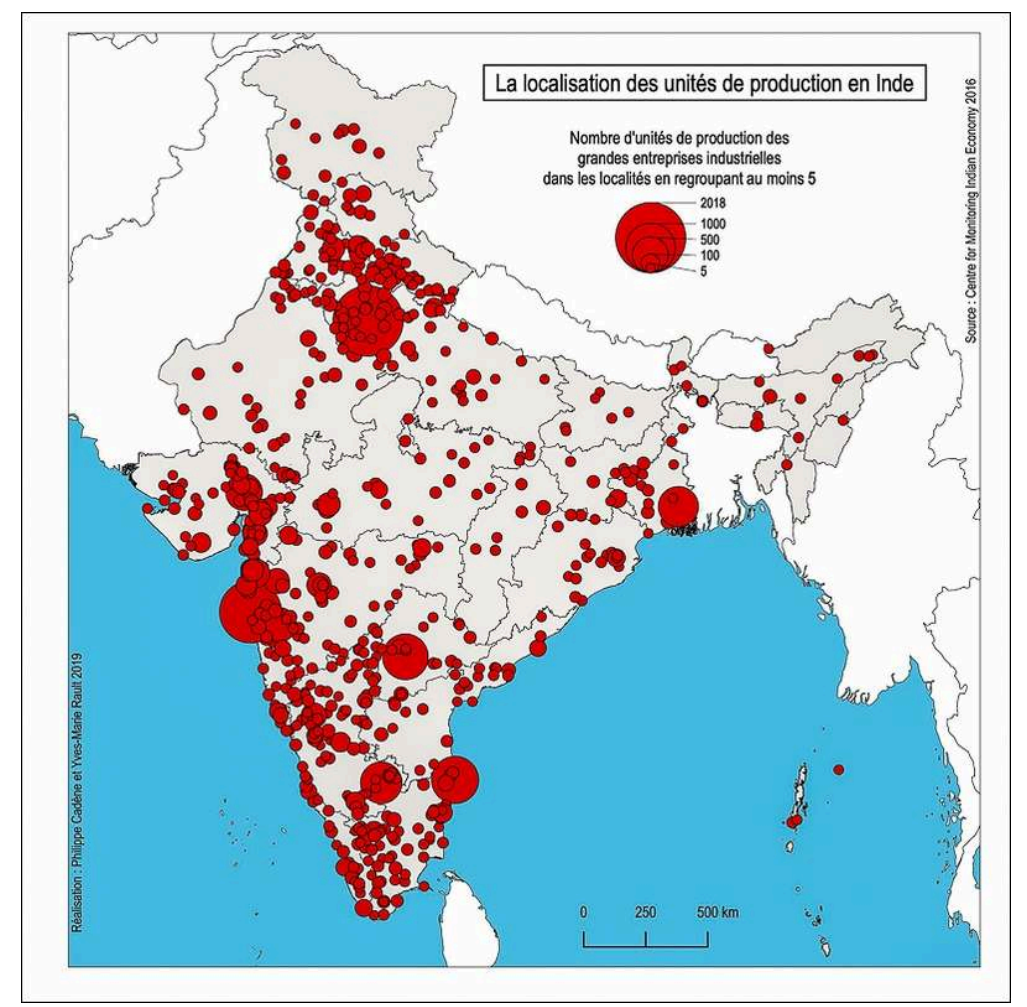

Source : Center for Monitoring Indian Economy, 2016 - Auteurs : P. Cadène et Y.-M. Rault, 2019.

L'observation de la carte montre que les plus grandes villes dominent toujours. Delhi prend désormais la première place. Avec 1945 unités de production appartenant aux grandes entreprises recensées par le CMIE, la capitale de l'Inde, désormais fortement marquée par un polycentrisme très diversifié, a manifestement renforcé au cours des deux dernières décennies la part des activités industrielles dans son économie. Cette vaste agglomération conserve un caractère de place décisionnelle et de place de service de haut niveau. Cependant, au gré de ses extensions, tout particulièrement de l'inclusion des cités industrielles anciennes de Ghaziabad et de Faridabad ou même de la construction de villes nouvelles comme Noida ou Gurgaon dans son aire métropolitaine, Delhi est devenue un centre de production essentiel dans le pays. La cartographie indique d'ailleurs une forte densité d'investissements industriels dans les territoires à la large périphérie ouest et nord de la capitale indienne, dans le nord-ouest du Rajasthan, en Haryana et au Punjab, dans le piémont himalayen de l'Uttarakhand et du nord-ouest de l'Uttar Pradesh.

Mumbai prend la seconde place pour les implantations des unités de production. Avec 1 578 usines établis sur le territoire de l'aire métropolitaine, le premier port du pays reste une grande cité industrielle à l'échelle de l'Inde. Mumbai est le centre décisionnel essentiel en matière économique, concentrant par exemple la presque totalité des activités bancaires et financières de haut niveau et une large part des services requérant des compétences fortes. La grande industrie qui y est implantée se caractérise d'ailleurs par de hautes valeurs ajoutées, dans la chimie fine, l'électronique, et encore la bijouterie. Les zones industrielles sont nombreuses, rejetées en périphérie (Imbach, 2011). Ce caractère de capitale économique qui marque toujours Mumbai, 
explique d'ailleurs une tendance à la délocalisation des industries loin à l'extérieur de l'agglomération, qui explique la position de la ville aujourd'hui. Les migrations des entreprises ont été observée dès le début des années 1980 à l'issue des grandes grèves des ouvriers du textile. Les prix de l'immobilier sont particulièrement élevés, et la circulation difficile en dépit des efforts municipaux. Les investisseurs choisissent donc de placer les activités productives dans d'autres villes du Maharastra, État dont Mumbai est la capitale. Deux villes phares pour les investissements productifs dans cet État sont Pune et Nashik. La ville de Silvassa, capitale du Territoire de l'Union Dadra and Nagar Haveli, est également un lieu d'investissements privilégié. Ancien comptoir portugais, ce petit territoire situé à la frontière entre les deux États du Maharastra et du Gujarat a longtemps bénéficié d'un statut de quasi-zone franche qui a su attirer les entreprises. Le Gujarat apparait par ailleurs comme un espace favorable aux investissements productifs. Le long du littoral de l'océan Indien, les villes industrielles sont nombreuses, les plus grandes étant Surat, Vapi et Vadodara. Ahmedabad enfin, capitale du Gujarat et déjà célèbre pour son industrie textile à la fin du $\mathrm{XIX}^{\mathrm{e}}$ siècle, compte aujourd'hui parmi les lieux les plus dynamiques du pays. Ses milieux d'affaires sont historiquement très liés à ceux de Mumbai, et l'agglomération, longtemps un grand centre textile, est désormais le siège d'un grand nombre d'industries chimiques et pharmaceutiques, installés dans les nombreux parcs établis par le gouvernement du Gujarat à la périphérie de la ville. Dans l'ensemble de cette région économique, les industries sont nombreuses dans des villes de moyenne ou de petite taille, ces dernières parvenant ou non à construire une dynamique de développement grâce à des communautés actives ( $C f$. Mukhopadhyaay et al., 2016 ; Denis et Zérah, 2017).

Les quatre grandes métropoles suivantes, Chennai, Hyderabad, Bangalore et Kolkata apparaissent relativement isolées au sein d'espaces où les unités de production se trouvent dispersées, souvent dans des villes de petites tailles. Dans le sud du Deccan, ces petites villes se trouvent toutefois en relativement grand nombre. Dans le nord, et particulièrement dans la partie est de la plaine indogangétique, où se situe Kolkata, bien peu de petites villes possèdent des unités de production en nombre et en taille suffisante pour être représentées sur la carte. Les activités de production industrielles, lorsqu'elles existent, sont de petite taille et ne parviennent pas à commercialiser leur produit au loin. Les entreprises ne réussissant à exporter que lorsqu'elles sont rassemblées au sein de districts industriels, qui ne se développent qu'en des lieux spécifiques dans le pays. Les deux États du Karnataka et du Kerala, ainsi que certaines parties du Tamil Nadu, concentrent un ensemble de villes petites et moyennes très actives sur le plan industriel. Ailleurs dans le pays, les petits centres industriels apparaissent souvent s'égrener de façon linéaire, suivant les axes de communication Ils forment des couloirs dans lesquels les productions sont souvent spécialisées dans un secteur particulier, comme le montrent les recherches conduites en Inde sur les clusters industriels ${ }^{15}$ (Cadène et Holmström, 1998 ; Das, 2005 ; Bercegol et Gowda, 2017). Enfin, d'autres petites villes industrielles se trouvent dispersées au sein du territoire, certaines se montrant particulièrement actives. Par contre, des espaces entiers restent à peu près exempts de centres productifs. Les régions du nord-est, du centre, et quelques États de la partie orientale de l'Inde apparaissent au contraire en marge des dynamiques industrielles, avec quelques centres comme Bhopal au Madhya Pradesh ou Bhubaneshwar dans l'Orissa, mais qui souffrent de l'absence d'un important tissu industriel local. 
Davantage encore que dans le cas de la carte présentant les sièges sociaux des grandes entreprises, la répartition des unités de production qui leur sont liées met en avant un très fort déséquilibre territorial en faveur de l'ouest de pays. Les deux plus grandes régions productives s'étendent au nord-ouest. La grande région de Delhi est constituée de la partie la plus occidentale de la plaine indogangétique. La grande région de Mumbai s'étend et s'élargit vers le nord de cette très grande agglomération. Ces ensembles sont les seuls à reproduire dans une certaine mesure les corridors industriels promus par le gouvernement, sans toutefois ne correspondre à un seul des projets avancés.

\section{La diffusion inégale du capital productif : grandes régions industrielles et réseaux logistiques}

Dans cette dernière partie, l'intention est de montrer combien, au-delà des phénomènes de concentration de l'industrie ou de son égrènement sur le territoire indien, des phénomènes de diffusion spatiale du capital productif à partir des grands centres urbains construisent des territoires industriels. Ces phénomènes sont observés à partir des mêmes données du CMIE utilisées précédemment, mais l'analyse s'inscrit cette fois, non pas à l'échelle des villes, grandes ou petites, mais à celles des 640 districts existant en Inde en 2011. Ceux-ci constituent une trame administrative datant souvent de la période précoloniale et organisent les services administratifs à l'intérieur des États fédérés. L'usage des données économiques et sociales recueillies ou organisées à ce niveau permet des analyses fines, pertinentes pour conduire une réflexion sur les territoires indiens (Chakravorty et Lall, 2007). Comme dans la partie précédentes, deux cartes ont été réalisées, mais cette fois, construites sur la base d'un calcul des densités des sièges sociaux d'entreprises ainsi que des unités de production à l'échelle des districts. Cette nouvelle grille d'analyse tend à effacer le phénomène de concentration de la production et à mettre en avant, au contraire, les phénomènes de diffusion de l'industrie sur le territoire. Les grandes régions de production apparaissent alors de façon marquée et, surtout, un étirement de la localisation des investissements le long des axes de transport peut être observé, permettant une intéressante réflexion sur le développement des corridors industriels. Afin de mieux visualiser ces phénomènes, les choix méthodologiques pour la réalisation cartographique ont conduit à ne conserver sur les deux cartes que les districts classés dans les quatre déciles supérieurs de l'ensemble statistique considéré. 
Illustration 5 - La densité de sièges sociaux industriels en Inde

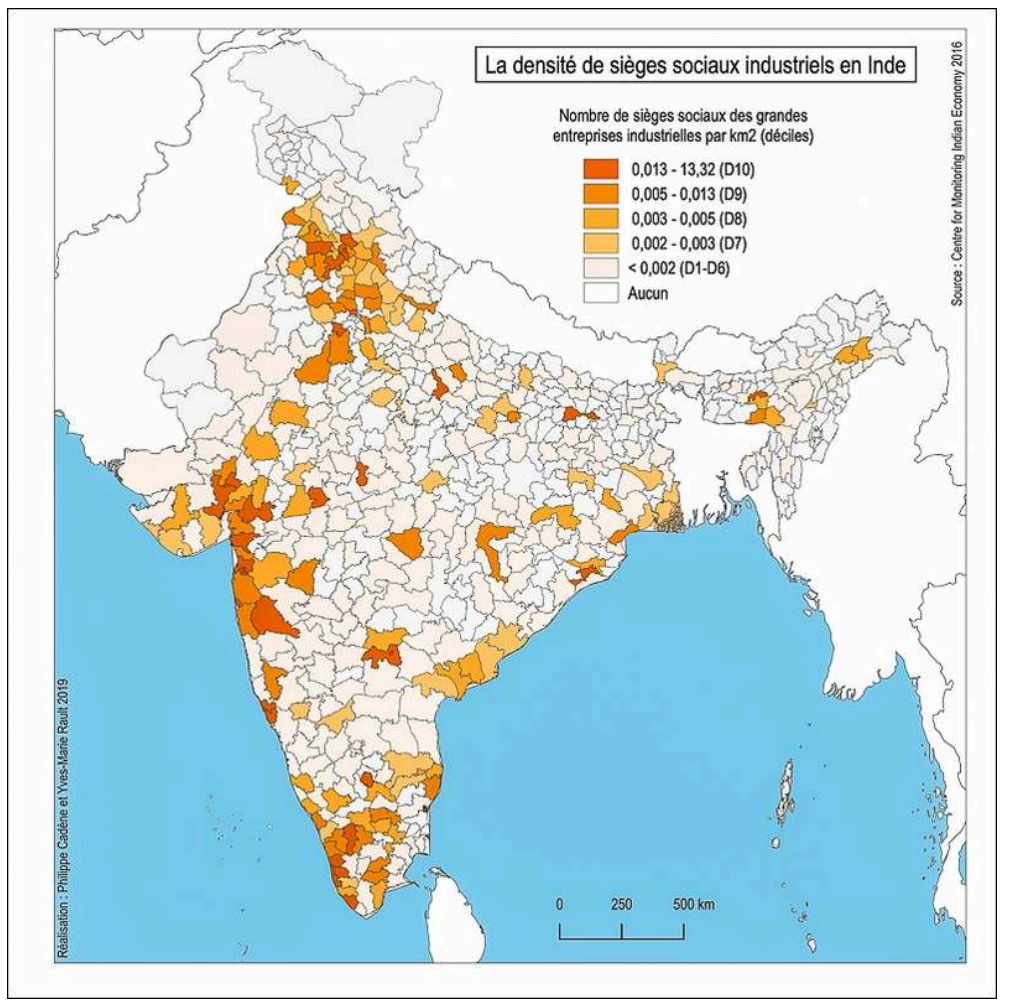

Source : Center for Monitoring Indian Economy, 2016 - Auteurs : P. Cadène et Y.-M. Rault, 2019.

Illustration 6 - La densité d'unités de production en Inde

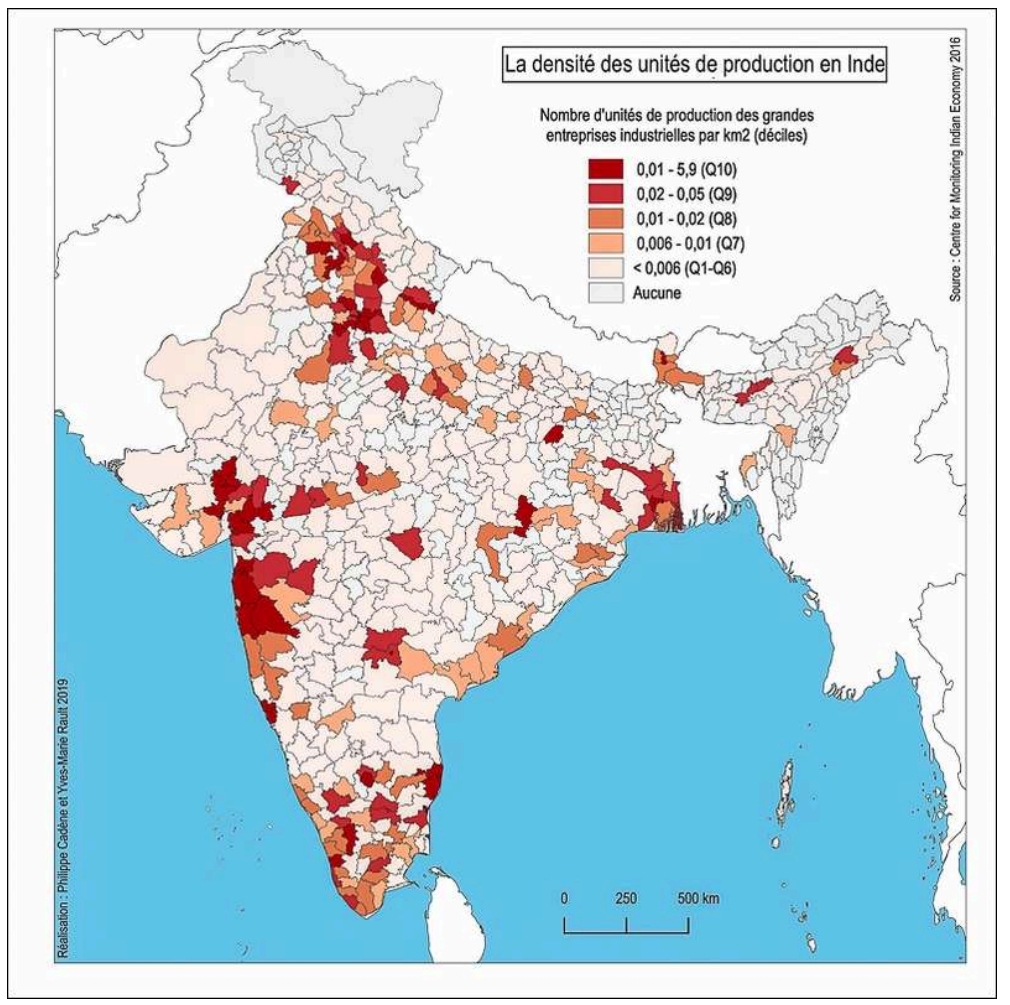

Source : Center for Monitoring Indian Economy, 2016 - Auteurs : P. Cadène et Y.-M. Rault, 2019. 


\section{Les grandes régions industrielles : le glissement vers l'ouest de la production} des sièges sociaux, l'autre celle des unités de production, confirme les déséquilibres territoriaux de la production industrielle par la présence de trois grandes régions productives dans le pays : l'une au nord incluant le Punjab et la grande région de Delhi, l'autre au centre ouest s'étendant sur le Gujarat et sur la partie occidentale du Maharashtra, une autre enfin dans le sud du pays, incluant surtout le Kerala et le Tamil Nadu.

Le glissement vers l'ouest de l'économie indienne, et tout particulièrement de l'économie industrielle, apparaissait déjà essentiel à partir des années 2000 dans l'analyse des données économiques et spatiales (Cadène, 2008). Elle s'affirme encore à la fin de cette décennie et dans les années 2010, mais la dynamique de la région de Kolkata marque désormais la géographie économique du pays (Cadène, 2015). Comme le montrent les travaux de Kundu et Varghese (2011), de Chakravorty (2012), et de Sanga et Shaban (2017), l'analyse des districts confirme l'importante force économique des régions industrialisées d'Inde de l'Ouest, et dans une moindre mesure d'Inde du Sud, où les sièges sociaux et les unités de production sont en grand nombre. Certains groupes de districts des États agricoles d'Inde du Nord, mais sous influence de la capitale Delhi, ont aussi connu un développement fort comme l'Haryana et le Punjab, alors que des États ayant longtemps bénéficié d'exemptions fiscales comme l'Himachal Pradesh ou l'Uttarakhand présentent de fortes densités dans certains de leurs districts, même s'ils ne semblent pas former de régions productives entièrement homogènes. La présente cartographie des districts, basée sur des données de 2016, conforte encore cette représentation du territoire indien, en montrant toutefois l'émergence de nouveaux territoires productifs. Elle met ainsi en lumière, à l'intérieur des agglomérations, des centres très denses, à l'instar du district de Mumbai Surburban, autour desquels sont situés des districts périphériques qui constituent des couronnes industrielles dont les densités régressent à mesure qu'on s'éloigne du centre. Ce schéma est par ailleurs bien davantage marqué pour les sièges sociaux que dans le cas des unités de production, généralement situées dans des zones moins denses. Il apparaît que les régions économiques en développement se construisent à partir d'un noyau métropolitain.

\section{La construction des corridors industriels par les investisseurs privés}

Outre la mise en avant de grandes régions industrielles, les deux cartes montrent une extension des investissements au-delà de ces dernières. Des alignements de clusters industriels dans lesquels se concentrent les entreprises sont clairement observables dans la carte présentant la densité des sièges sociaux. Ils se manifestent plus fortement encore avec la carte fondée sur la densité des unités de production, qui montre une extension territoriale des implantations par rapport à la première carte.

Dans l'État du Rajasthan, un ensemble de districts où les grandes entreprises sont fortement représentées s'étend vers le sud, à partir de Delhi jusqu'au Gujarat. Là commence la puissante région industrielle de la côte ouest dominé par Mumbai, située au sud de celle-ci. L'existence du corridor Delhi-Mumbai soutenu par l'État central 
apparaît ainsi manifeste, les districts industrialisés du Rajasthan faisant le lien entre les deux principaux centres économiques du pays. La connaissance du terrain permet par ailleurs de savoir que le district de Rajsamand, le seul district du corridor laissé en blanc par la méthode de cartographie choisie prenant seulement en compte les unités spatiales classées dans les quatre déciles supérieurs, a connu depuis les années 1980 le développement d'un immense cluster industriel spécialisé dans la taille du marbre et du granit.

Un corridor entre Bengaluru et Chennai apparaît également de façon très nette sur les deux cartes, les districts qui le composent associant d'ailleurs une implantation de grandes entreprises, sièges sociaux et unités de production qui explique leur présence sur les cartes, avec un foisonnement d'entreprises de taille moyenne ou petite, rassemblées en cluster. Il semble fortement correspondre au tracé proposé par les pouvoirs publics.

Un autre corridor projeté par l'État, le corridor allant de Visakhapatnam à Chennai, semble se dessiner. Sa partie nord est présente sur les deux cartes, du district de Visakhapatnam où se situe la cité portuaire éponyme aux deux districts littoraux voisins de Khrisna et de Guntur. Ces deux districts abritent les villes en plein essor de Vijayawada et de Guntur, à proximité desquelles, Amaravati, la future capitale du nouvel État de l'Andhra Pradesh, est en construction ${ }^{16}$. Sa partie sud apparaît sur la carte présentant les unités de production avec les deux districts de Tiruvallur, dans le Tamil Nadu, au nord de Chennai, et de Nellore, sur le littoral de l'Andhra Pradesh. Cette dernière carte montre aussi l'existence d'un autre corridor qui relie les deux villes de Vijayawada et de Guntur à Hyderabad, puissante capitale du nouvel État du Telangana, qui commandait l'ensemble de cette région avant la division de l'Andhra Pradesh en deux entités séparées. À partir d'Hyderabad, un autre corridor est présent sur la carte, descendant vers le sud à travers l'État du Karnataka pour attendre la ville de Mysore, longtemps connue pour ses soies artisanales, qui est aujourd'hui un centre touristique, mais aussi une cité industrielle dynamique, aux activités diversifiées, dont des entreprises de hautes technologies sont en plein essor. Ces deux derniers corridors, de Vijayawada-Guntur à Hyderabad et d'Hyderabad à Mysore, ne sont en rien des projets du gouvernement central, mais plutôt le résultat de liens tissés par les milieux d'affaires locaux avec les États fédérés dans lesquels ils se trouvent. Cela explique certaines implantations d'entreprises, à la recherche d'avantages administratifs et de bénéfices offerts par diverses formes d'externalités.

D'autres corridors de ce type apparaissent sur les cartes. Deux se trouvent dans le sud du Deccan. Un corridor industriel s'étend le long de la côte du Kerala, intégrant les districts de Kozhikode, d'Ernakulam avec le port de Kochi et de Thiruvananthapuram, où se trouve la capitale de l'État. Un autre traverse le Tamil Nadu du nord au sud, observable sur la carte présentant la densité des unités de production. Au nord, à l'extérieur de l'État, se trouve la grande métropole de Bangalore, tandis qu'au Tamil Nadu le corridor passe par les districts de Salem, de Tiruchirappalli, de Madurai, jusqu'à celui de Kanniyakumari, à la pointe sud de la péninsule. La grande industrie est toutefois présente dans la totalité des districts au sud de l'État, lequel bénéficie par ailleurs d'un très grand nombre de clusters productifs rassemblant des entreprises moyennes et petites. Dans la partie centrale de l'Inde, se remarque un corridor partant du cœur industriel du Gujarat et s'étendant vers l'est, passant par le district d'Indore pour atteindre ceux de Bhopal, avec la ville du même nom, et de Raisen, avec la ville de 
Sanchi. À l'est du pays, un ensemble de districts apparaissent sur la carte, dans l'État de Chhattisgarh, au nord de l'État de l'Orissa, ainsi que dans le Jharkhand. Il s'agit d'espaces où l'extraction minière s'accompagne de la présence d'industries lourdes, sans toutefois produire une richesse capable d'engager la région dans un développement véritable. Un dernier corridor est observable dans les territoires $d u$ nord-est, situés à l'est du Bangladesh. Il s'étend sur un ensemble de districts de l'Assam, le long de la vallée du Brahmapoutre dans laquelle se trouve les deux villes de Guwahati et de Dibrugarh. Manufactures de thé et usines de transformation pétrolière sont les principales industries dans cette région assez reculée de l'Inde.

Des cinq corridors industriels projetés dans la stratégie de développement indienne, trois apparaissent donc dans les cartes de densité réalisées à partir des implantations des grandes entreprises industrielles, tandis que deux sont absents. Une partie du corridor en projet reliant Amritsar à Kolkata (ADKIC), ne connaît pas un niveau de développement industriel suffisant pour apparaître sur les cartes dans sa partie qui va de l'est de Delhi à Kolkata. Dans le nord-ouest du pays, le territoire des États du Punjab et l'Haryana, auxquels s'ajoutent des districts adjacents, constituent une puissante région industrielle liée au corridor Delhi-Mumbai. La partie suivante du corridor projeté, qui s'étend le long de la plaine indogangétique sur une distance d'environ $1500 \mathrm{~km}$, est caractérisée par des activités artisanales et par la présence de petites et moyennes entreprises fortement liées au milieu rural et agricole. La grande industrie se limite aux plus grandes villes: Kanpur, Lucknow, Varanasi, Patna. L'économie de plupart des districts de la plaine reste fortement marquée par l'agriculture. Il en va de même pour le corridor Mumbai-Bengaluru (MBEC), absent des cartes. L'agriculture est forte à peu près partout. Il existe aussi de nombreuses industries petites et moyennes parsemées sur le territoire de l'État du Karnataka. Seul se distingue le district de Dharwad, connu pour son usine d'automobiles Tata et ses entreprises de sous-traitance mécanique qui s'y sont développées.

Il apparaît donc clairement que les stratégies de développement territorial du gouvernement indien, associant les acteurs privés à l'action publique et encourageant particulièrement les investissements étrangers, accompagnent une dynamique économique qui tend depuis un peu plus de vingt ans à intégrer des espaces économiques n'ayant jusqu'alors que peu de liens, ou ne concernant que certains secteurs particuliers. Ainsi, les corridors industriels en développement sur le territoire indien sont plus nombreux que ceux projetés et encouragés par l'État central. Par contre, ces derniers se trouvent dans des situations variées. Les stratégies mises en place à l'échelle nationale sont reprises de façons diverses selon les États régionaux, certains soutenant la politique des corridors, d'autres mettant en avant d'autres priorités. Les politiques des pouvoirs publics, du centre ou des États fédérés, ne sont pas forcément coordonnées et sont loin de toujours répondre aux déterminants économiques et sociaux qui orientent les stratégies des milieux entrepreneuriaux. Ces processus entrainent de fortes inégalités territoriales dans la répartition du capital productif. Les enquêtes réalisées en divers lieux du pays montrent par ailleurs que les choix du type de localisation des entreprises dépendent des caractéristiques de ces dernières. Certaines nécessitent la proximité des institutions financières, de services de haut niveau, d'une main d'œuvre fortement qualifiée. D'autres cherchent au contraire un accès facilité aux matières premières, des coûts du foncier limités et une force de travail abondante et bon marché (Roberts, 2016). Le choix du lieu, par contre, peut dépendre de déterminants liés à l'histoire des entreprises et celles des personnes qui 
les dirigent. Les entreprises obéissent certes à des logiques dictées par des réseaux, parfois internationaux, dans lesquels elles sont intégrés, mais elles sont aussi issues d'environnement économique, social, et politique particulier où elles sont insérées et auxquelles elles doivent s'adapter le cas échéant (Hite, 2003 ; Kloosterman, 2010).

\section{Conclusion} elle montre également, comme il en a été fait l'hypothèse au début de la recherche, que les corridors planifiés par l'État indien, incluant la construction d'infrastructures de transport et de nombreux projets de développement, ne sont pas tous accompagnés par les investisseurs. Ces derniers privilégient en effet d'autres corridors industriels qui apparaissent à l'analyse des cartes présentant l'implantation des grandes entreprises.

téngie de développement territorial menée par l'État apparaît toutefois en cohérence avec les politiques publiques antérieures qui visaient à faciliter la mise en relation des régions économiques du territoire indien héritées de la période coloniale. L'Inde a conduit un effort considérable à partir de la fin des années 1990 pour construire un réseau autoroutier jusqu'alors inexistant. En moins de dix ans, l'État a su mettre en place le "quadrilatère doré ", un lien autoroutier entre les cinq principaux pôles urbains que sont Delhi, Kolkata, Chennai, Bangalore et Mumbai ${ }^{17}$. Le long de ces axes, les aéroports ont été modernisés. Tout un ensemble d'investissements dans les infrastructures de transport et communication ont également été réalisés. De nombreux projets visant à l'amélioration des transports ferrés ont également été annoncés, suivant peu ou prou les trajets autoroutiers, mais sont eux encore loin d'être aboutis.

es corridors programmés par l'État reprennent ce quadrilatère, ne s'en écartant que pour y inclure la région du Punjab et de l'Haryana à l'ouest de Delhi, ou pour exclure le segment entre Visakhapatnam et Kolkata, un espace encore en retrait par rapport au développement du pays, où probablement les investissements privés sont les plus difficiles à attirer.

Les localisations des entreprises industrielles suivent manifestement d'autres logiques que celles consistant à se calquer sur les projets conduits par le gouvernement. Elles privilégient tout d'abord les très grandes villes, accentuant la domination économique des plus grands centres urbains. Le capital productif semble ainsi avant tout à la recherche des externalités positives offertes par les grandes villes. Cette tendance apparait massivement si l'on prend en compte l'implantation des sièges sociaux, qui se situent massivement dans les principales métropoles du pays. Elles continuent à jouer un rôle dans la localisation des unités de production, bien que celles-ci se répartissent davantage sur le territoire, particulièrement dans certaines régions, contribuant le plus souvent à construire des axes, plus ou moins structurés.

Apparaissent ainsi des corridors industriels qui offrent une partie des externalités rencontrées dans les grandes concentrations urbaines. Outre l'atout de situer une entreprise sur un axe de transport fréquenté, l'intérêt d'une pareille localisation réside en effet dans l'efficacité d'un fonctionnement en réseau offrant la proximité relative d'un ensemble de services, voire d'un marché, et permettant en outre de sélectionner des espaces dans lesquels les coûts du foncier ou de la main d'œuvre sont relativement faibles. L'implantation le long d'un corridor peut alors apparaître comme une solution 
raisonnable. Par contre, ces stratégies industrielles conduisent à accentuer les inégalités économiques au sein du territoire indien.

Les dynamiques mises en avant montrent ainsi que l'État conserve un rôle important dans le développement du territoire. Cependant, le processus de libéralisation économique permet aux entrepreneurs de conduire leurs propres stratégies. Alors que les États de la fédération voient s'accroitre leurs compétences face à l'État central, les pouvoirs publics et les milieux entrepreneuriaux s'engagent dans un dialogue qui conduit à construire une forme particulière de gouvernance, inédite en Inde à ce jour. Le développement des corridors est une illustration de cette situation nouvelle.

\section{BIBLIOGRAPHIE}

Abélès M, 2008. Anthropologie de la globalisation. Paris, Payot, Petite Bibliothèque.

Arrighi G., 2001. Global Capitalism and the Persistence of the North-South Divide. Science \& Society, 65,4 .

de Bercegol, R. Gowda S., 2017. Development on the urban fringe: the prosperity of Kartarpur, of Small cluster-town in the Punjab. In Denis E., Zérah M.-H. (ed.), Subaltern Urbanization in India. An introduction to ordinary towns' dynamics. Delhi, Springer India.

Boillot J.-J., 2016. L'économie de l'Inde (3édition). Paris, La Découverte.

Brenner N., Peck J., Theodore N., 2010. Variegated neoliberalization: Geographies, modalities, pathways. Global networks, vol. 10, $\mathrm{n}^{\circ} 2$.

Brunner H.-P., 2013. What is Economic Corridor Development and What Can It Achieve in Asia's Subregions? ADB Working Paper Series on Regional Economic Integration, $\mathrm{n}^{\circ} 117$.

Cadène Ph., Holmström M. (ed.) 1998. Decentralized production in India. Industrial districts, flexible specialization and employment. New-Delhi, Sage Publications.

Cadène Ph., 2008. Atlas de l'Inde. Paris, Autrement.

Cadène Ph., 2012. Les métropoles secondaires indiennes dans la mondialisation. Territoires de l'urbain en Asie du sud-est : métropolisations en mode mineur. CNRS Editions.

Cadène Ph., 2015. Différenciation et intégration du territoire indien. In Cadène P. et Dumortier B., L'Inde : une géographie. Paris, Armand Colin.

Cadène Ph., 2017a. Tourism Development in the Thar Desert, Rajasthan. In Lavie E. and Marshall A. (ed.), Oases and Globalisation, Springer International Publishing.

Cadène Ph., 2017b. La construction d'une vaste région économique au nord-ouest de l'Inde : le corridor de développement Delhi-Mumbai. Bulletin de l'Association de Géographes Français, $\mathrm{n}^{\circ} 1$.

Castells M., 1996. The rise of the network society. Chichester et Malden, Wiley-Blackwell.

Chakravorty S., et Lall S. V., 2007. Made in India: the economic geography and political economy of industrialization. New Delhi et New York, Oxford University Press. 
Chakravorty S., 2012. Regional Development in India: Paradigms Lost in a Period of Great Change. Eurasian Geography and Economics, vol. 53, $\mathrm{n}^{\circ} 1$.

Chaudhuri B., 2015. La libéralisation de l'économie indienne, ses étapes et ses effets. In Cadène Ph., Dumortier B., L'Inde. Une géographie, Paris, Colin.

Corbridge S., Harriss J., 2000. Reinventing India: Liberalization, Hindu nationalism and popular democracy. Hoboken New Jersey, John Wiley \& Sons.

Corbridge S., Williams G., Srivastava M.K., Véron R., 2005. Seeing the state: governance and governmentality in India. Cambridge, University Press Cambridge.

Datta A., 2015. New urban utopias of postcolonial India: 'Entrepreneurial urbanization' in Dholera smart city, Gujarat. Dialogues in Human Geography, vol. 5, $n^{\circ} 1$.

Das K., 2005. Indian Industrial Clusters. London, Routledge.

Debrie J., et Comtois C., 2010. Une relecture du concept de corridors de transport. Illustration comparée Europe/Amérique du Nord. Les Cahiers scientifiques du transport, $\mathrm{n}^{\circ} 58$.

Denis E., et Zérah M.-H. (ed.), 2017. Subaltern Urbanisation in India. Delhi, Springer India.

Dey I., Ray C. S. B., 2011. Negotiating Rights: The Case of a Special Economic Zone. In Chaudhury S.B., Dey I., Sustainability of Rights after Globalisation, Delhi, Sage.

Dey I., 2012. Emerging spaces and labour relations in neo-liberal India: A review essay. Policies and Practices, $\mathrm{n}^{\circ} 49$.

Dey I., Grappi G. 2015. Beyond Zoning: India's Corridors of “Development” And New Frontiers of Capital. South Atlantic Quarterly, vol. 114, $\mathrm{n}^{\circ} 1$.

Durand-Dastès F. et al., 1995. Monde indien. In Mutin G., Durand-Dastès F. (ed.), Afrique du Nord, Moyen-Orient, Monde indien. Belin.

Edginton D., Fernandez A., Hoshino C. (ed.), 2001. New Regional Development Paradigms. United Nations Centre for Regional Development, Greenwood Press.

Florida R. L., 2005. Cities and the creative class. New York, Routledge.

Ghani E., Kerr W. R., O'Connell S., 2014. Spatial Determinants of Entrepreneurship in India. Regional Studies, vol. $48, \mathrm{n}^{\circ} 6$.

Harvey D. W., 2008. Géographie de la domination. Paris, les Prairies ordinaires.

Hite J. M., 2003. Patterns of multidimensionality among embedded network ties: a typology of relational embeddedness in emerging entrepreneurial firms. Strategic organization, $\mathrm{n}^{\circ} 1$.

Imbach R., 2011. Vers une " global city region » ? Stratégies économiques, déploiement spatial et politiques d'accompagnement à Mumbai. Métropoles, $\mathrm{n}^{\circ} 9$.

Kamath L., Zachariah Y., 2015. Impact of JNNURM and UIDSSMT/IHSDP Programmes on Infrastructure and Governance Outcomes in Cities/Towns in India. A Review of the State of Knowledge. Mumbai, TISS Working Paper $n^{\circ} 7$.

Kennedy L., 2015. Stratégies spatiales de l'État en Inde. Le réétalonnage de l'État au travers de la politique des zones franches. EchoGéo, $\mathrm{n}^{\circ} 32$.

Khan S., Taraporevala P., Zérah M.-H., 2018. Les villes intelligentes indiennes : défis communs et diversification des trajectoires. Flux, vol. 4, $\mathrm{n}^{\circ} 114$. 
Kloosterman R. C., 2010. Matching opportunities with resources: A framework for analyzing (migrant) entrepreneurship from a mixed embeddedness perspective. Entrepreneurship and Regional Development, vol. 22, $\mathrm{n}^{\circ} 1$.

Kumar P., 2014. Industrial Corridors and Policy Imperatives for Their Success. IOSR Journal of Business and Management, vol. 16, n 6, p. 44-51.

Kundu A., Varghese K., 2010. Regional Inequality and "Inclusive Growth" in India under Globalization: Identification of Lagging States for Strategic Intervention. New Delhi, Oxfam India Working Paper Series.

Manna G. C., 2010. Current status of industrial statistics in India: Strengths and weaknesses. Economic and Political Weekly, $\mathrm{n}^{\circ}$ 67-76.

McCartney M., 2009. Political economy, growth and liberalisation in India, 1991-2008. New York, Routledge.

Milbert I., 2007. Mondialisation, gouvernance et développement. In Cadène Ph. (ed.), La mondialisation. L'intégration des pays en développement, Paris, SEDES.

Milbert I., 2017. Dynamiques publiques et privées autour des smart cities en Inde. Bulletin de l'Association de Géographes Français [En ligne], vol. 94, n 1, p. 5-22. http:// journals.openedition.org/bagf/280 ; DOI : 10.4000/bagf.280

Mukhopadhyaay P., Zerah M.-H., Samanda G. et al., 2016. Understanding India's urban frontier: what is behind the emergence of census towns in India? The World Bank.

Mukhopadhyay C., 2018. Is the Delhi Mumbai Industrial Corridor (DMIC) an Emerging 'Megaregion' in India? Planning Theory \& Practice, vol. 19, $\mathrm{n}^{\circ} 2$.

Nadvi K., Schmitz H., 1994, Industrial Clusters in Less Developed Countries: Review of Experiences and Research Agenda. Brighton, Institute of Development Studies, Discussion Paper n 339.

Rodrigue J.-P., Notteboom T., 2010. Foreland-based regionalization: Integrating intermediate hubs with port hinterlands. Research in Transportation Economics, $\mathrm{n}^{\circ} 27$.

Roberts M., 2016. Identifying the economic potential of Indian districts. The World Bank.

Rothermund D., 2002. An Economic History of India. Abingdon-on-Thames, Routledge.

Sanga P., Shaban A., 2017. Regional Divergence and Inequalities in India. Economic \& Political Weekly, vol. 52, $\mathrm{n}^{\circ} 1$.

Sassen S., 1996. La ville globale: New york, Londres, Tokyo. Paris, Descartes \& Cie.

Sassen S. (ed.), 2002. Global networks, linked cities. New York, Routledge.

Scott A. J. (ed.), 2001. Global city-regions: trends, theory, policy. Cambridge et New York, Oxford University Press.

Sharma N. K., 2009. Special Economic Zones: Socioeconomic Implications. Economic and Political Weekly, vol. 24, $\mathrm{n}^{\circ} 20$.

Sheppard E., 2012. Trade, globalization and uneven development : Entanglements of geographical political economy. Progress in Human Geography, vol. 36, $\mathrm{n}^{\circ} 1$.

Sivaramakrishnan K. C., 2011. Re-visioning Indian cities: The urban renewal mission. Delhi, SAGE Publications India.

Srivastava P., 2011. Regional Corridors Development in Regional Cooperation. Asian Development Bank Economics Working Paper Series, $\mathrm{n}^{\circ} 258$. 
Vakulabharanam V., De R., 2016. Growth and Distribution Regimes in India after Independence. The Round Table, vol. 105, nº 6, p. 621-640.

Veltz P., 1997. Mondialisation, villes et territoires : l'économie d'archipel. Paris: PUF.

Wallerstein I., 2000. Globalization or the Age of Transition? A Long-Term View of the Trajectory of the World-System. International Sociology, vol. 15, $n^{\circ} 2$.

\section{NOTES}

1. Nous utilisons tout au long cet article le terme de globalisation en accord avec Marc Abélès, qui le choisit afin de montrer l'existence d'une rupture avec les phases précédentes de la mondialisation. L'intention est de « rendre compte du niveau d'intégration et d'interconnexion qui est désormais atteint (depuis les années 1990) et qui se traduit par la perception empirique chez les individus, par-delà leurs attaches territoriales et leurs identités culturelles, d'une appartenance à un monde » (Abélès, 2008, p. 8).

2. En 2007, 600 villes génèrent $60 \%$ du PIB mondial selon le rapport du McKinsey Global Institute, Urban world: Mapping the economic power of cities, mars 2011.

3. À partir du milieu des années 1990, souvent sous la pression des partis nationalistes hindous ou des partis régionalistes, certains noms de villes, en particulier les plus grandes, ont été changés, à la recherche d'une origine parfois incertaine. Ainsi Bombay fut nommée Mumbai, Calcutta Kolkata, Madras Chennai et Bangalore Bengaluru.

4. Tout particulièrement à Delhi NCR, au Gujarat, au Maharashtra, au Rajasthan, en Uttar Pradesh, mais également en Andhra Pradesh et au Karnataka.

5. Des données publiques existent et sont mêmes plus étendues, comme celles du Annual Survey of Industries, du Follow-up Establishment Surveys of the Economic Census, les census-cum-surveys of smallscale industries, le Central Statistical Organisation's Index of Industrial Production ou le ponctuel Economic Census. Mais elles souffrent d'un manque de standardisation et de rigueur méthodologique (Manna, 2010), conduisant de nombreux chercheurs à utiliser les chiffres fournis par le CMIE

6. Cf. Industrial Corridor. Make In India (mai 2019) [En ligne]. http://www.makeinindia.com/ article/-/v/delhi-mumbai-industrial-corrid-1 (consulté le 4/09/2019).

7. Une composante du JNURM orientée vers le développement des petites villes (l'UIDSSMT) n'a pas été effective. Seules les plus grandes villes ont bénéficié du programme. Cf. Sivaramakrishnan, 2011 et Kamath et Zachariah, 2015.

8. Cf. Kumar, 2014; https://dipp.gov.in/sites/default/files/order_DMIC_PITF_02January2017.pdf; Industrial Corridors [En ligne], Department for Promotion of Industry and Internal Trade, MoCI | GoI. https://dipp.gov.in/programmes-and-schemes/infrastructure/industrial-corridors

9. Cf. DMICDC. An Overview [En ligne]. https://dmicdc.com/about-DMICDC

10. Du nord au sud: Manesar-Bawal-Investment Region, Haryana; Dadri-NoidaGhaziabad Investment Region, Uttar Pradesh; Khushkhera-Bhiwadi-Neemrana Investment Region, Rajasthan; Jodhpur Pali Marwar Industrial Area, Rajasthan; Pithampur-Dhar-Mhow Investment Region, Madhya Pradesh; Dholera Special Investment Region, Gujarat; Shendra-Bidkin Industrial Area, Aurangabad, Maharashtra ; Dighi Port Industrial Area, Maharashtra.

11. Cf. Road Ministry Sets Record Target of Constructing $40 \mathrm{KM}$ Highways Per Day To Build 12,000 KM Network In FY20. (s.d.) [En ligne]. https://swarajyamag.com/insta/road-ministry-setsrecord-target-of-constructing-40-km-highways-per-day-to-build-12000-km-network-in-fy20 (consulté le 1/09/2019). 
12. Pour un bilan de la participation du secteur privé aux investissements dans les infrastructures à la fin de la décennie 2000, voir: Gupta P., Gupta R., Netzer N., 2009. Building India. Accelerating Infrastructure Projects. McKinsey India. Pour le Department of Economic Affairs, Ministry of Finance, voir: Government of India, 2015. Report of the Committee on Revisiting and Revitalising Public Private Partnership Mode of Infrastructure. November.

13. 12th Five Year Plan (2012-2017), Planning Commission, http://planningcommission.gov.in/ plans/planrel/12thplan/pdf/12fyp_vol2.pdf

14. Les données chiffrées concernant la population des plus grandes villes de l'Inde sont celles fournies par le recensement de l'Inde de 2011. D'autres documents publiés depuis proposent des chiffres bien plus élevés, autour de 20 millions d'habitants par exemple pour Delhi et Mumbai. Cf. United Nations New York, Department of Economic and Social Affairs Population Division, 2019. World Urbanization Prospects The 2018 Revision.

15. Le terme cluster a été introduit dans la littérature de langue anglaise pour décrire des ensembles de PME dans les pays émergents ou en développement qui possédait les caractères des districts industriels, mais se différenciaient par un faible niveau technique ou une absence de formalisation des relations interentreprises ( $C f$. Nadvi et Schmitz, 1994).

16. L'État d'Andhra Pradesh a été divisé en 2014 pour créer un nouvel État, le Telangana avec Hyderabad pour capitale, et l'Andhra Pradesh qui s'étend désormais seulement sur la partie littorale de l'ancien État et dont la future capitale sera Amaravati.

17. À ce quadrilatère autoroutier, clé pour le développement du pays, ont été ajoutés deux axes de transport transversaux, sans être accompagnés d'autres projets d'investissements. Un axe nord-sud commence à Srinagar dans l'État du Jammu-andCachemire et aboutit à Kanyakumari au Tamil Nadu. Un autre axe de transport ouestest lie Porbandar au Gujarat à Silchar en Assam.

\section{RÉSUMÉS}

L'État indien s'est engagé depuis les années 2000 dans une politique de développement territorial orientant les investissements le long de corridors où sont implantés des infrastructures de transport modernisées et des zones spécialisées pour recevoir des entreprises. Cinq « corridors industriels ou économiques » ont été officiellement identifiés par les décideurs, reliant les plus grandes agglomérations du pays. L'analyse des localisations d'entreprises montre que, dans un contexte de croissance forte et soutenue, une polarisation de l'économie du pays se poursuit au profit des principales métropoles et le long de corridors, construisant de grandes régions où se diffusent les investissements et les activités de production. Les logiques d'implantation des entreprises obéissent à des déterminants complexes. Elles sont éloignées des stratégies de développement de l'État et s'effectuent dans la cadre d'une gouvernance qui affirme le rôle des pouvoirs publics régionaux et des acteurs privés dans la structuration du territoire indien.

Since the early 2000s, the Indian State has engaged into a territorial development policy that supports investments along corridors with modernized transport infrastructures and adapted areas to accommodate businesses. Five « industrial or economic corridors » have been formally identified by the government, connecting the country's largest cities. The analysis of business locations shows that, in a context of strong and sustained growth, the country's productive 
capital is concentrating in the main cities and along corridors, shaping large regions capturing investments and industrial activities. The location of companies is determined by a set of complex factors. Their logics are distinct from the State development strategies and are framed in a governance which strengthens the role of regional public authorities and private actors in the spatial structuration of India.

\section{INDEX}

Mots-clés : corridor, métropole, industrie, capital, Inde

Keywords : corridor, metropolis, industry, capital, India

Thèmes : Sur le Champ - Sur le Terrain

\section{AUTEURS}

\section{PHILIPPE CADÈNE}

Philippe Cadène, philippe.cadene@univ-paris-diderot.fr, est Professeur à l'Université Paris Diderot et chercheur à l'UMR CESSMA. Il a récemment publié :

- Cadène P., 2017. La construction d'une vaste région économique au nord-ouest de l'Inde : le corridor de développement Delhi-Mumbai. Bulletin de L'Association des géographes français [En ligne], vol. 94, $\mathrm{n}^{\circ}$ 1, p. 40-61. http://journals.openedition.org/bagf/302 ; DOI : 10.4000/bagf.302 - Cadène P., 2017. Tourism Development in the Thar Desert, Rajasthan. In Lavie E., Marshall A. (ed.), Oases and Globalisation. Springer International Publishing.

- Cadène P., 2018. Asie du Sud. In Mareï N., Richard Y. (ed.), Dictionnaire de la régionalisation du monde. Paris, Atlande.

\section{YVES-MARIE RAULT}

Yves-Marie Rault, yves.marie.rault@gmail.com, est doctorant à l'Université Paris Diderot, UMR CESMA. Il a récemment publié :

- Rault Y.-M., Mathews S., Cebeillac A., 2018. The commercialisation of shopping malls in India: from 'creating' to 'meeting' demand. Bulletin de l'Association de géographes français [En ligne], vol. 95, n 1, p. 61-78. http://journals.openedition.org/bagf/2656 ; DOI : 10.4000/bagf.2656 - Rault Y.-M., 2017. Classes moyennes et construction d'une modernité indienne. Le cas des 'shopping malls' à Delhi. Bulletin de l'Association de géographes français [En ligne], vol. 94, $\mathrm{n}^{\circ} 1$, p. 77-94. http://journals.openedition.org/bagf/321 ; DOI : 10.4000/bagf.321

- Cebeillac A., Rault Y.-M., 2016. Contribution of geotagged Twitter data in the study of a social group's activity space. The case of the upper middle class in Delhi, India. Netcom. Réseaux, communication et territoires [En ligne], vol. 30, n³-4, p. 231-248. http://journals.openedition.org/ netcom/2529 ; DOI : 10.4000/netcom.2529 